\title{
Principais Considerações da Extrusão Termoplástica de Alimentos
}

Danilo M. Santos, Andrea L. Bukzem, Nayara D. Coutinho, José L. R. Ascheri \& Diego P. R. Ascheri

Por sua versatilidade, custo relativamente baixo, alta produtividade e ser considerado como tecnologia limpa, o processo de extrusão termoplástica vem sendo utilizado para a elaboração de novos produtos alimentares, realizada praticamente em uma única operação. Cada produto é obtido por meio de condições controladas que garante a qualidade do alimento extrusado. Por isso, é de extrema importância conhecer o fundamento do processo de extrusão, o extrusor e suas partes principais e os fatores que interferem e interagem na qualidade do produto. O presente trabalho faz uma revisão de alguns aspectos importantes do processo de extrusão termoplástica em alimentos.

Palavras-chave: processamento de alimentos, alimentos extrudados, parâmetros do processo de extrusão.

For their versatility, relatively low cost, high productivity and be regarded as clean technology, the thermoplastic extrusion process has been used for the development of new food products, carried out almost in a single operation. Each product is obtained through controlled conditions to ensure the quality of the extruded food. It is therefore of outmost importance to know the foundation of the extrusion process, the extruder and its main parts and the factors that interfere and interact in product quality. This paper reviews some aspects of the process for thermoplastic extrusion in food.

Keywords: processing of food, food extruder, parameters of extrusion process. 


\section{Introdução}

O processo de extrusão termoplástica de alimentos é uma tecnologia que teve origem na indústria de plásticos e já conta com mais de 70 anos. Extrusores mono roscas foram utilizados em 1935 para dar forma a macarrões e cereais pré-cozidos, mas somente nos anos 40 foram desenvolvidos extrusores com grandes motores elétricos, para cozimento, com o propósito de preparar snacks ${ }^{1}$. Desde então, o processamento de alimentos por extrusão termoplástica vem ganhando destaque e expansão na indústria de alimentos por ser uma importante técnica que, além de aumentar a variedade de alimentos processados, apresenta muitas vantagens quando comparado a outros sistemas de processamento de alimentos, como versatilidade, custo relativamente baixo, alta produtividade e, por representar um processo ambientalmente seguro, é uma tecnologia catalogada como limpa ${ }^{2,3,4}$.

Em virtude da sua versatilidade operacional e suas múltiplas funções, esta tecnologia tem encontrado um vasto campo de aplicações, seja para a produção de alimentos para o consumo humano ou para o consumo animal. A extrusão de alimentos permite maior facilidade na produção de misturas alimentícias destinadas ao consumo humano, produzindo uma variedade de produtos, tais como: alimentos infantis, proteínas vegetais texturizadas, bebidas em pó instantâneas, amidos modificados para uso industrial, cereais pré-cozidos, snacks, farinhas instantâneas e amidos prégelatinizados utilizados na formulação de sopas de preparo rápido, molhos semiprocessados, produtos de confeitaria e outros ${ }^{5,6,7,8}$.

A extrusão termoplástica consiste em um tratamento térmico a uma temperatura elevada durante curto tempo (em inglês high temperature sorth time, HTST) ${ }^{9}$. O princípio básico deste processo é converter um material sólido em fluído pela aplicação de calor e trabalho mecânico e comprimi-lo através de uma matriz ${ }^{10}$. O processo promove a gelatinização do amido, a desnaturação e reorientação das proteínas, a inativação enzimática, a destruição de substâncias tóxicas tais como os inibidores das proteases e a diminuição da contagem microbiana para formar um produto de características físicas e geométricas pré-determinadas ${ }^{11}$. Além disso, proporciona a hidratação de amidos e proteínas, homogeneização, cisalhamento, fundimento de gorduras, plastificação e expansão da estrutura alimentar ${ }^{5,12}$.

A extrusão é um processo extremamente versátil e o equipamento pode se comportar como trocador de calor devido às trocas térmicas envolvendo as paredes do cilindro, a rosca e o material. Desempenha, também, a função de reator químico de processamento de alimentos em condições de altas temperaturas (até $250{ }^{\circ} \mathrm{C}$ ), altas pressões (até $25 \mathrm{MPa}$ ), num tempo de residência curto (1 a 2 min). Nessas condições, durante o processo, a desnaturação de proteínas causa a abertura das estruturas terciárias e quaternárias resultando na quebra e rearranjo das pontes de hidrogênio e dissulfetos, permitindo a plastificação e a formação de texturas desejáveis ${ }^{10,13}$. No amido, o processo de extrusão promove a gelatinização, criando subprodutos com propriedades diferenciadas.

\section{EXTRUSOR}

O extrusor (Figura 1) é composto basicamente por cinco partes principais, sendo um sistema de alimentação (funil de alimentação), parafuso com mecanismo de acionamento (motor e redutores de velocidade), cilindro, matriz e mecanismo de corte ${ }^{4}$.

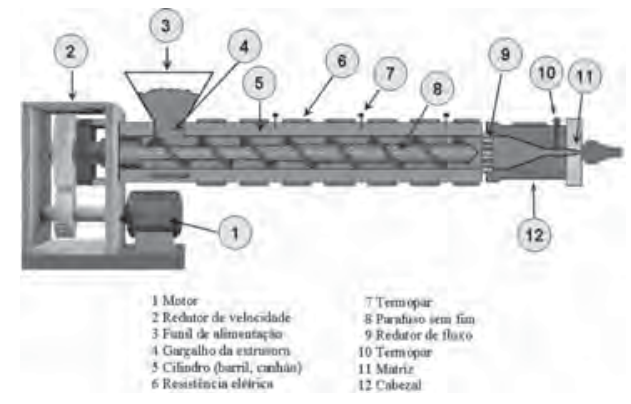

Figura 1. Esquema simplificado de um extrusor mono-rosca.

O sistema de alimentação é constituído por um recipiente que recebe o material. Este sistema pode conter um parafuso de alimentação localizado verticalmente ou horizontalmente. Com intuito de permitir um melhor fluxo de material, o sistema de alimentação pode ser equipado com agitador. O modelo do sistema de alimentação é um ponto crítico, pois é necessário que haja um suprimento adequado do material, a fim de permitir o bom funcionamento do extrusor e evitar flutuações no processo de cocção e nas características dos produtos obtidos ${ }^{14,15}$.

$\mathrm{O}$ parafuso apresenta-se como parte central e 
principal do equipamento, possuindo em sua divisão as zonas de alimentação, transição e alta pressão (zona de cocção) de acordo com a Figura 2.

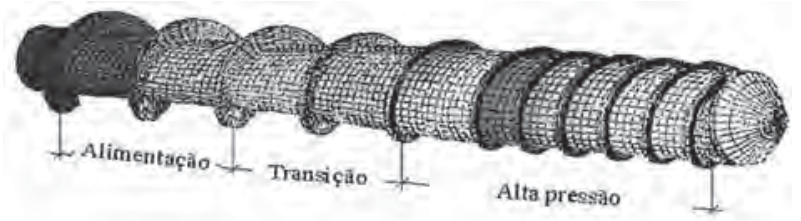

Figura 2. Esquema de um parafuso simples simulando o fluxo de calor nas diferentes zonas de alimentação, transição e alta pressão.

O extrusor pode possuir um ou dois parafusos sem fim, ou roscas, que podem ser mono ou dupla rosca, giratórios, firmemente encaixados dentro de um cilindro para alimentar, coccionar e pressionar o material ${ }^{16}$. Os de mono-rosca são simples e de menor custo e têm atuação limitada às matérias-primas com umidade variando de 10 a 30\% e baixo teor de lipídeos, enquanto que as extrusoras de dupla rosca são altamente versáteis, pois operam com umidade mais elevada (até 90\%) com maior teor de lipídeos, velocidade variada do parafuso e o seu mecanismo de transporte é independente das forças de atrito $^{13}$. A Figura 3 mostra exemplos de extrusores mono e dupla-rosca, respectivamente.

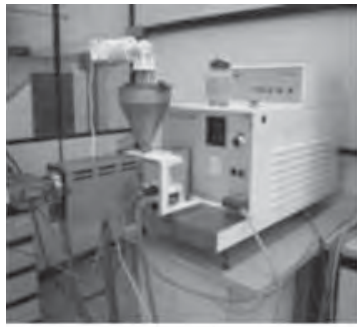

(a)

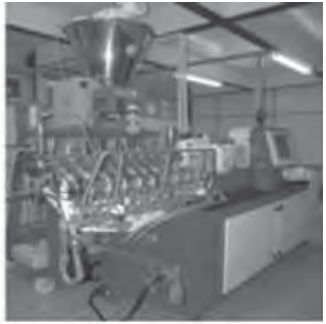

(b)
Figura 3. (a) extrusor mono-rosca marca BRABENDER (modelo 20DN) e (b) extrusor dupla-rosca marca Clextral, modelo Evolun HT25.

A rotação do parafuso conduz o material em direção à matriz revirando e pressionando-o contra as paredes do cilindro o que promove as transformações físicas e químicas no material. O aquecimento e controle da temperatura em cada uma das zonas do extrusor são muito importantes, pois são uns dos fatores principais que determinará o grau de transformação dos componentes da mistura a ser extrusadas. O aquecimento pode ser realizado através de resistências elétricas (Figura 4a) e o controle da temperatura pode ser feito por meio de serpentinas de ar comprimido ou camisas de água fria incluídas nas resistências elétricas (Figura 4b). Muitos cilindros são equipados com sensores de pressão e temperatura, os termopares, para controle do processo ${ }^{4,16}$.

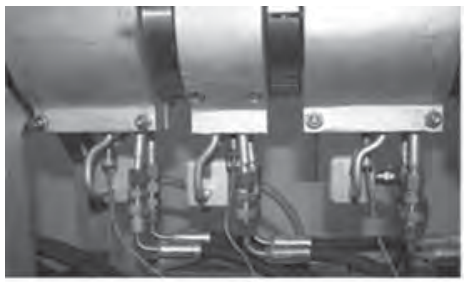

(a)

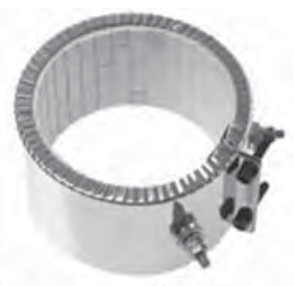

(b)
Figura 4. Resistências elétricas tubulares: a) mostrando as instalações dos termopares e de sistema de esfriamento e b) vista interna de uma resistência elétrica com núcleo cerâmico e dispositivo para a inclusão de termopares.

O final do cilindro é equipado com a matriz (Figura 5) que, geralmente é constituída de vários orifícios possuindo a função de moldar o produto no formato desejado e trabalhar como um redutor de fluxo, aumentando a pressão na zona de cozimento do extrusor ${ }^{17}$. Na saída da matriz pode ser instalado um mecanismo para cortar o produto extrusado. Este mecanismo deve ser constituído de um motor cortador de velocidade para propiciar a obtenção de extrusados de comprimento uniforme ${ }^{1}$.

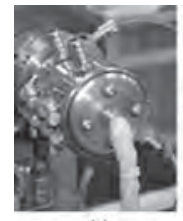

(a)

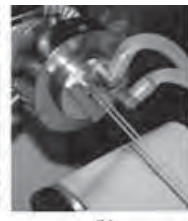

(b)

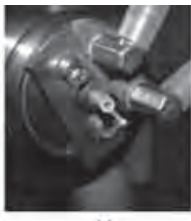

(c)

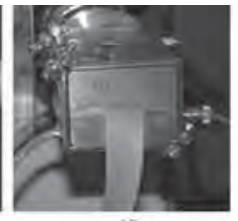

(d)
Figura 5. Matriz de extrusão: a) de um orifício, b) de dois orifícios, c) de um orifício para a elaboração de macarrão e, c) para a produção de laminados.

\section{PROCESSO DE EXTRUSÃO}

O princípio fundamental do processo de extrusão é converter um material sólido em um fluido pela aplicação de calor e trabalho mecânico e comprimi-lo através de uma matriz, promovendo assim a termoplastificação do mesmo. O parafuso ao girar conduz o material na direção de uma matriz aplicando-lhe, portanto, um cisalhamento e transformando-o numa massa uniforme ${ }^{13}$. O processo de 
extrusão compreende as etapas de pré-extrusão, extrusão e pós-extrusão. A pré-extrusão inclui a preparação dos ingredientes e sua mistura em proporção adequada. Após a mistura, o material é transportado para ser condicionado a um conteúdo apropriado de umidade e em algumas extrusoras, como a extrusora Clextral, modelo Evolun HT25, apresentada na Figura 3b, a matéria-prima é condicionada a umidade na própria máquina durante a etapa de extrusão ${ }^{15,16}$.

Na etapa de extrusão, a matéria-prima é introduzida no equipamento através do alimentador, sendo impulsionada pelo(s) parafuso(s) em direção à matriz. À medida que o produto atravessa as diferentes zonas de extrusão (de alimentação, de transição e de alta pressão), ocorre aumento gradativo do atrito mecânico, provocado por modificações da geometria do parafuso e abertura da matriz. Em consequiência, aumentam também a pressão e a temperatura, ocorrendo o cozimento do produto. A pósextrusão inclui a secagem dos extrusados para umidades inferiores a $10 \%$, o resfriamento e, em alguns casos, a aplicação de sabores e temperos ${ }^{10,16}$.

\section{Fatores que Interferem na Qualidade dos Produtos Extrusados}

Os fatores que mais influenciam a natureza dos produtos extrusados são: características da matéria-prima e as condições de operação do extrusor ${ }^{18}$.

\section{CARACTERÍSTICAS DA MATÉRIA-PRIMA}

As propriedades da matéria-prima têm importante influência na textura e coloração do produto. Os fatores mais importantes são tipo, teor de umidade, estado físico e composição química, particularmente a quantidade e tipo de amido, proteínas, gorduras e açúcares. O pH do material umidificado também interfere ${ }^{18}$.

A composição da matéria-prima, seu teor de umidade e tamanho de suas partículas influenciam a viscosidade do produto no extrusor. A viscosidade é um fator crucial, que determina as condições de operação do extrusor, e, portanto, a qualidade do produto final. Diferentes tipos de matérias-primas geram produtos completamente diferentes quando algumas condições de operações do extrusor são usadas. Isto decorre dos diferentes tipos e teores de amido e de proteínas, teores de umidade e outros ingredientes adicionados (por exemplo, óleo e emulsificantes), o que resulta em diferentes viscosidades e características de fluxo. Semelhantemente, a adição de ácidos para ajuste do $\mathrm{pH}$ causa mudanças na gelatinização do amido e no desdobramento de moléculas de proteínas. Diferenças no conteúdo de açúcares e no pH também produzem variação de cor devido a Reação de Maillard ${ }^{8,10,18}$.

Partículas pequenas, como as farinhas, são hidratadas mais facilmente e cozidas mais rapidamente que partículas maiores, alterando também a qualidade do produto. A gelatinização do amido normalmente causa aumento na viscosidade, mas em extrusãococção, o intenso cisalhamento também pode quebrar as macromoléculas em unidades menores, resultando na redução da viscosidade e consequente formação de dextrinas. Em alguns casos isto pode ser benéfico e em outros pode ser excesso de taxa de cisalhamento ${ }^{18}$.

Vários autores estudaram o efeito da formulação de misturas de alimentos nas características dos produtos extrusados. A inclusão de ingredientes no material amiláceo a ser extrusado influencia as características físico-químicas do produto final, podendo diminuir a gelatinização do amido por não oferecerem material viscoso suficiente ${ }^{19}$. Carvalho et al. $^{20}$ estudando a influência da mistura de farinha de trigo, arroz e banana, e Fernandes et al. ${ }^{21}$, em canjica e soja, observaram que a formulação das farinhas do produto a ser extrusado influencia a gelatinização do amido. Também afirmaram que quanto maior a proporção de material não-amiláceo, menor o grau de gelatinização do amido do produto extrusado em decorrência da diminuição da proporção de amido.

\section{CONDIÇÕES DE OPERAÇÃO DO EXTRUSOR}

Embora o processo de extrusão seja basicamente uma simples operação tecnológica, seu controle é complicado. Isto decorre das inúmeras variáveis envolvidas no processo e do fato de que a influência da maioria delas sobre o processo não é conhecida. O controle das condições do processo de extrusão é uma tarefa fundamental, pois não somente permite a obtenção de produtos com características tecnológicas variadas, mas também melhora a eficiência e economia da operação ${ }^{15}$. Segundo El-Dash ${ }^{15}$, 
Guerreiro $^{1}$, Silva ${ }^{4}$, Ascheri e Carvalho ${ }^{16}$, os parâmetros de operação mais importantes no extrusor são umidade de processamento da matéria-prima, temperatura do cilindro, configuração da matriz, configuração do parafuso e velocidade do parafuso ${ }^{22}$.

\section{UMIDADE DE PROCESSAMENTO}

O aumento do conteúdo de água acarreta normalmente o decréscimo na viscosidade do produto, que tem como primeiro efeito o abaixamento da geração de calor e, como segundo efeito, o decréscimo da queda de pressão do produto na saída da matriz ${ }^{1}$. A umidade tem um efeito pronunciado nas características dos extrusados. Geralmente, baixos valores de umidade estão relacionados ao maior grau de dextrinização do amido e menor viscosidade da pasta a frio ${ }^{15}$. Segundo Onwulata et al. ${ }^{23}$ a umidade exerce grande influência na qualidade do produto, pois esta produzirá maior ou menor taxa de cisalhamento, sendo que se for muito baixo pode afetar a estrutura celular e a fragilidade dos produtos expandidos. De acordo com o percentual de água será formada uma massa no interior do extrusor com diferentes características de viscosidade, o que implica em diferenças na expansão ${ }^{8}$.

Para se obter produtos com características diferentes, os extrusados devem possuir umidades iniciais distintas, como por exemplo para se obter salgadinhos expandidos, como os elaborados de grits de milho, a umidade deverá estar entre 15 e 16\%, aproximadamente. Já para produtos diferentes, como os denominados 'pellets" ou snacks de terceira geração, a umidade de processamento estará entre 24 a 26\%, isto por que neste produto, não se deseja a expansão, apenas um pré-cozimento, a expansão acontecerá após secagem e posterior fritura ou submissão ao forno de microndas $22,24,25$. O aumento da umidade diminui o grau de degradação do amido durante o aquecimento e cisalhamento devido à redução do estresse, que promove redução da viscosidade ${ }^{26}$.

\section{TEMPERATURA DO CILINDRO}

A temperatura utilizada no processo de extrusão exerce papel importante nas mudanças das propriedades físico-químicas dos produtos extrusados. Lawton et al. ${ }^{27}$ consideraram, além da umidade inicial da matériaprima, a temperatura como a variável de maior efeito na gelatinização do amido. Van Lengerich ${ }^{26}$ observou que amidos degradados são compostos de moléculas com baixo peso molecular em decorrência da dextrinização do amido. Esse fenômeno, usualmente ocorre em temperaturas de extrusão maiores do que $160{ }^{\circ} \mathrm{C}^{19}$. Entretanto, Li et al. ${ }^{28}$ observaram maior grau de gelatinização do amido de milho quando extrusado a $130{ }^{\circ} \mathrm{C}$. Sacchetti et al. ${ }^{29}$ reforçaram essa observação, porém usando amido de arroz extrusado em temperatura de $120^{\circ} \mathrm{C}$.

\section{CONFIGURAÇÃO DO PARAFUSO}

A função, a eficiência e a capacidade de um extrusor dependem, em larga extensão, do desenho do parafuso e de seu revestimento, os quais governam o mecanismo de fluxo. O parafuso é provavelmente a parte mais importante do extrusor, pois este governa não somente o grau de cocção e a gelatinização, mas também a característica de qualidade do produto final ${ }^{4}$.

Na configuração de um extrusor, os elementos do parafuso, restritores de fluxo, podem ser considerados para otimizar o grau de cozimento do material. Cada máquina possui suas próprias características, porém o barril e o parafuso requerem específica configuração para um determinado produto (Figura 6). Uma inadequada configuração diminui as possibilidades de controle de alguns parâmetros como velocidade do parafuso e temperatura do barril ${ }^{4,16}$. O tempo de residência do produto no extrusor é uma função do modelo do parafuso e de sua velocidade de rotação. Normalmente, o tempo de residência varia de 60 a 270 segundos $^{30}$.

\section{VELOCIDADE DE ROTAÇÃO DO PARAFUSO}

A uma maior velocidade no parafuso, observase maior taxa de cisalhamento. Isto implica em um maior grau de cozimento do material, sendo que, como consequência de uma maior velocidade, o tempo de resistência diminui devendo-se observar os níveis ótimos destes parâmetros a fim de se conseguir qualidade no produto final ${ }^{19}$.

\section{CONFIGURAÇÃO DA MATRIZ}

A configuração e desenho da matriz são fundamentais na qualidade do produto final. As taxas de cisalhamento podem ser alteradas dramaticamente pela mudança de uma matriz. Ao mudar uma matriz simples por outra 
múltipla se observa que o fluxo de material aumenta, em função das mudanças ${ }^{16}$.

A intensidade de pressão dentro do cilindro depende, em parte, do tamanho da matriz. Se o cilindro for completamente aberto na matriz final, não haverá pressão e o extrusor simplesmente agirá como um parafuso transportador. Inversamente, se a matriz final for completamente fechada, a pressão aumentará. Entre estes dois extremos, o tamanho da matriz afeta grandemente o desempenho do extrusor ${ }^{5,10}$.

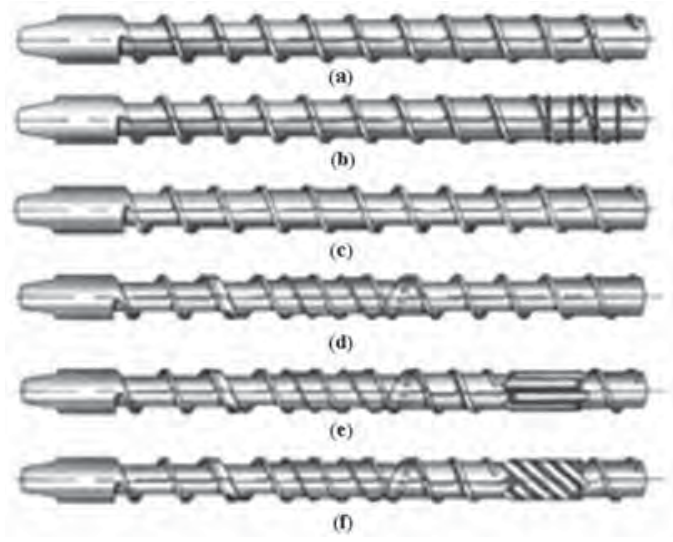

Figura 6. Tipos de parafusos sem fim de extrusão: a) básico; b) básico com pinos misturadores;, c) de duas etapas com aeração; d) de duas etapas com barreira tipo "DSB"; e) com barreira tipo "DSBM" e com elemento cisalhador tipo Maddock; f) com barreira tipo "DSBM-T" e com elemento cisalhador espiral tipo Maddock.

\section{Considerações Finais}

Pode-se considerar que o processo de extrusão é uma ferramenta para o desenvolvimento de produtos. Segundo a sua aplicação podem ser processados grãos inteiros, moídos grossos, farinhas, misturas de farinhas para fins alimentícios, tanto para consumo humano ou em rações, quanto para animais domésticos ou para criação, inclusive na aquicultura, rações para peixes e camarões, etc. $\mathrm{Na}$ indústria em geral os benefícios dos extrusados podem ser aproveitados na indústria da mineração, extração de petróleo, têxtil, etc. Tudo isto, somado ao adequado uso das matérias-primas, a configuração do equipamento e suas diferentes nuances aplicativas bem como manejo dos parâmetros, definem o tipo de produto. A versatilidade que oferece este processo dependerá da criatividade e imaginação do produtor e na sua procura por novas estruturas e inovações em alimentos.

\section{Referências Bibliográficas}

1. Guerreiro, L. Produtos Extrusados para Consumo Humano, Animal e Industrial. Rio de Janeiro, 2007, 25 p. Dossiê Técnico - SBRT.

2. Carvalho, R. V. Formulações de snacks de terceira geração por extrusão: caracterização texturométrica e microestrutural. 2000. 89 p. Dissertação (Mestrado em Ciência de Alimentos). Universidade Federal de Lavras, Lavras.

3. Guy, R. Extrusión de los alimentos. Zaragoza: Acribia, 2001. 208 p.

4. Silva, E. M. M. Produção de macarrão pré-cozido à base de farinha mista de arroz integral e milho para celíacos utilizando o processo de extrusão. 2007, 118p. Dissertação(Mestrado em Ciência e Tecnologia de Alimentos). Universidade Federal Rural do Riode Janeiro, Rio de Janeiro.

5. Fellows, P. J. Food Processing Technol.: principles and practice. 2 ed. Cambridge: Midway Technology Limited, 2000. 575 p.

6. Baik, B.; Powers, J.; Nguyen, L. T. Extrusion of regular and waxy barley flours for production of expanded cereals. Cereal Chem., St. Paul, v. 81, n. 1, p. 94-99, 2004.

7. Souza, M. L.; Menezes, H. C. Extrusão de misturas de castanha do Brasil com mandioca. Ciênc. Tecnol. Aliment., Campinas, v. 28, n. 2, p. 451 - 462, 2008.

8. Vernaza, M. G.; Chang, Y. K.; Stell, C. J. Efeito do teor de maracujá e da umidade e temperatura de extrusão no desenvolvimento de cereal matinal funcional orgânico. Braz. J. Food Technol., v.12, n.2, p. 145-154, 2009.

9. Fellows, P. J. Tecnologia do processamento de alimentos: princípios e prática. $2^{\mathrm{a}}$ ed., Porto Alegre: Artmed, 2006, 602p.

10. Borba, A. M. Efeito de alguns parâmetros operacionais nas características físicas, físico-químicas e funcionais de extrusados da farinha de batata-doce. 2005. 115 p. Dissertação (Mestrado em Ciência e Tecnologia de Alimentos) - Universidade de SãoPaulo, Piracicaba.

11. Sebio, L. Efeito de alguns Parâmetros Operacionais de Extrusão nas Propriedades Físico-químicas da farinha de inhame (Dioscorearotundata). 1996. 106p. Dissertação (Mestrado) Faculdade de Engenharia de Alimentos, Universidade Estadual de Campinas, Campinas.

12. Ascheri, J. L. R., Carvalho, C. W. P. de Produção de extrusados doces. Série Documentos Embrapa Ctaa. , p.1 - 18, 2004.

13. Sebio, L. Desenvolvimento de plástico biodegradável a base de amido de milho e gelatina pelo processo de extrusão: Avaliação das propriedades mecânicas, térmicas e de barreira. 2003. 179 p. Tese (Doutorado em Tecnologia de Alimentos), Universidade Estadual de Campinas - Faculdade de Engenharia de Alimentos, Campinas, São Paulo.

14. Sunderland, R. Continuous breading production by extrusion cooking. Cereal Foods World, St. Paul, v.38, n.9, p.685-688, 1993.

15. El-Dash, A. A. Aplication and control of thermoplastic extrusion 
of cereals for food and industrial uses. In: Cereals a renewable resurce: theory and practice. American Association of Cereal Chemists, St. Paul, p.165-216, 1981.

16. Ascheri, J. L. R.; Carvalho, C. W. P. Processo de extrusão de alimentos: aspectos tecnológicos para o desenvolvimento e produção de alimentos para consumo humano. Rio de Janeiro, 2008, 82 p. Apostila do Curso de extrusão termoplástica de alimentos - EMBRAPA Agroindústria de Alimentos.

17. Borges, J. T. S. Borges, J. T. S.: Elaboração do macarrão précozido à base de farinha integral de quinoa (Chenopodiumquinoa, Willd) e farinha de arroz (Oryzia sativa, L.) utilizando processo de extrusão termoplástica. 2002. 116 p. Dissertação (Mestrado em Ciência e Tecnologia dos Alimentos) - Universidade Federal Rural do Rio de Janeiro/ UFRRJ, Rio de Janeiro.

18. Fellows, P. Extrusion. In: Fellows, P. Food processing technol.: principles and practive. Cambridge: Woodhead Publishing, 2002. cap.14, p.294-308.

19. Ascheri, D. P. R.; Andrade, C. T.; Carvalho, C. W. P.; Ascheri, J. L. R. Obtenção de farinhas mistas pré-gelatinizadas a partir de arroz e bagaço de jabuticaba: efeito das variáveis de extrusão nas propriedades de pasta. Boletim do Centro de Pesquisas e Processamento de Alimentos, Curitiba, v. 24, n. 1, p. 115-144, 2006.

20. Carvalho, R. V.; Ascheri, J. L. R.; Vidal, J. Efeito dos parâmetros de extrusão nas propriedades físicas de pellets (3G) de misturas de farinhas de trigo, arroz e banana. Ciênc Agrotec, Lavras, v.26, n.5, p.1006-18, 2002.

21. Fernandes, M. S.; Wang, S. H.; Ascheri, J. L. R.; Oliveira, M. F.; Costa, S. A. J. Produtos extrusados expandidos de misturas de canjiquinha e soja para uso como petiscos. Pesquisa Agropecuária Brasileira, v. 37, n. 10, p. 1495-1501, 2002.

22. Souza, V.F, Ortiz, J. A. R., Nascimento, E. M. G. C. Do, Ascheri, J. L. R. Pasting properties of expanded extrudate and pellets from corn flour and rice flour. Braz. J. Food Technol. (Impresso). , v.14, p.106 - 114, 2011.

23. Onwulata, C. I.; Smith, P. W.; Konstance, R. P.; Holsinger, V. H. Incorporation of whey products in extruded corn, potato or rice snacks. Food Research International, v.34, p.679-687, 2001.

24. Ascheri, J. L. R., Carvalho, C. W. P., Spehar, C. R. Extrusão do amaranto no desenvolvimento de produtos: caracterização físicoquímica. Série Documentos Embrapa Ctaa. , p.1 - 32, 2004.
25. Ascheri, J. L. R., Carvalho, C. W. P., Matsuura, F. C. A. U. Elaboração de pellets de harina de raspa de mandioca por extrusión termoplástica (escala piloto e industrial). Alimentaria , v.XXXVII, p.101 - 106, 2000.

26. Van Den Einde, R. M.; Akkermans, C.; Van Der Goot, A. J.; Boom, R. M. Molecular breakdown of corn starch by thermal and mechanical effects. Carbohydr. Polym., v.56, p.415-422, 2004.

27. Lawton, B. J.; Henderson, G. A.; Derlatska, E. J. The effects of extruder variables on the gelatinization of corn starch. Can. J. Chem. Eng., v. 50, n. 4, p. 168-172, 1972.

28. Li, P. X.; Campanella, O. H.; Hardacre, A. K. Using an in-line slitdie viscometer to study the effects of extrusion parameters on corn melt rheology. Cereal Chem., v. 81, n. 1, p. 70-76, 2004.

29. Sacchetti G.; Pinnavaia, G. G.; Guidolin, E.; Dalla Rosa, M. Effects of extrusion temperature and feed composition on the functional, physical and sensory properties of chestnut and rice flour-based snack like products. Food Research International, v. 37, p. 527-534, 2004.

30. Harper, J. M. Extrusion processing of food. Food Technol., v.32, n.7, p.67-72, Jul. 1978.

Danilo M. Santos', Andrea L. Bukzem', Nayara D. Coutinho', José L. R. Ascheri² \& Diego P. R. Ascheri $^{1^{*}}$

${ }^{1}$ Universidade Estadual de Goiás, Unidade Universitária de Ciências Exatas e Tecnológicas. Br 153, n. 3105- Fazenda Barreiro do Meio, C. P. 459, Anápolis/GO, Brasil.

${ }^{2}$ Embrapa Agroindústria de Alimentos, Av. das Américas, 29501, Guaratiba, CEP 23020-470, Rio de Janeiro/RJ.

*e-mail: ascheridpr@uol.com.br 